\title{
PACIFIC: SiPM Readout ASIC for LHCb Upgrade
}

H. Chanal, A. Comerma, D. Gascón, S. Gómez, X. Han, J. Mazorra*, N. Pillet, R. Vandaele on behalf of the LHCb SciFi group *IFIC (Valencia) and PI (Heidelberg)

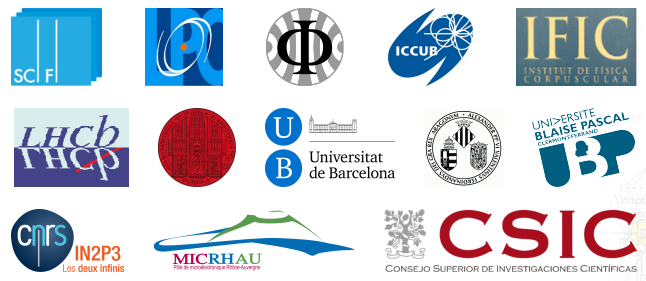

IEEE NSS/MIC - Strasbourg - $2^{\text {nd }}$ November 2016

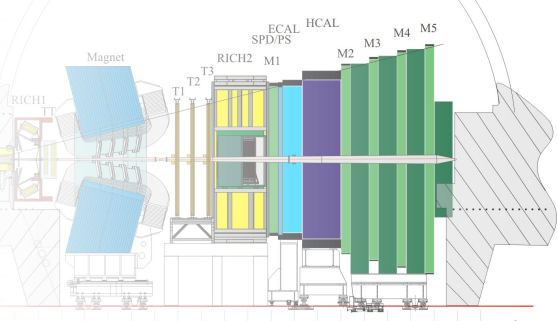




\section{Outline}

(1) Introduction

(2) PACIFIC

- Preamplifier

- Shaper

- Integrator

- Digitizer

(3) Prototypes

- PACIFICrO $/ 1$

- PACIFICr2/3

(4) Outlook 


\section{Introduction: $\mathrm{LHCb}$}

PACIFIC:

SiPM Readout

Introduction

PACIFIC

Preamplifier

Shaper

Integrator

Digitize

Prototypes

PACIFICrO/

$\mathrm{PACIFICr} 2 / 3$

Outlook
- Physics measurements limited by $1 \mathrm{MHz}$ hardware trigger.

- Upgrade: increased luminosity, $40 \mathrm{MHz}$ trigger at front-end.

- New detector for T1-T3 the Scintillating Fibre Tracker.
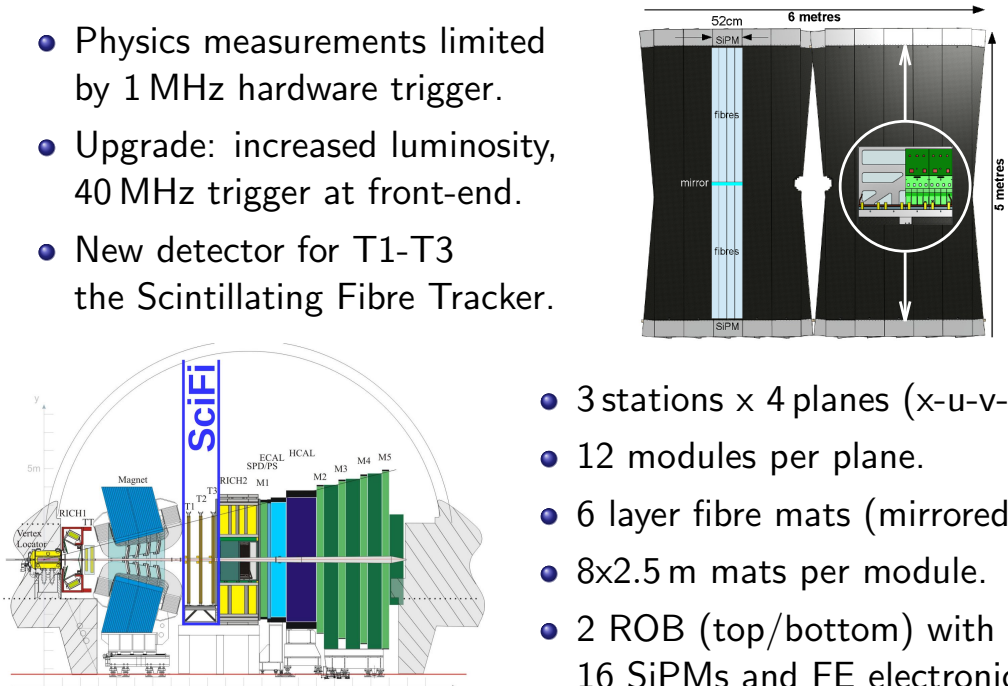

- 3 stations $x 4$ planes $(\mathrm{x}-\mathrm{u}-\mathrm{v}-\mathrm{x})$.

- 12 modules per plane.

- 6 layer fibre mats (mirrored).

- 8x2.5 m mats per module.

- 2 ROB (top/bottom) with 16 SiPMs and FE electronics. 


\section{PACIFIC}

PACIFIC: SiPM Readout

Introduction

\section{PACIFIC}

Preamplifier

Shaper

Integrator

Digitizer

Prototypes

$\mathrm{PACIFICrO} /$

PACIFICr2

Outlook
- Readout with 64 channel in CMOS $130 \mathrm{~nm}($ IBM $\rightarrow$ TSMC).

- Current mode input for direct anode connection.

- Configurable non-linear digital output serialized.

- Fast shaping to minimize spillover.

- Signal integration to overcome low photostatistics using dual interleaved system to avoid dead time.

- Single photoelectron detection capability.

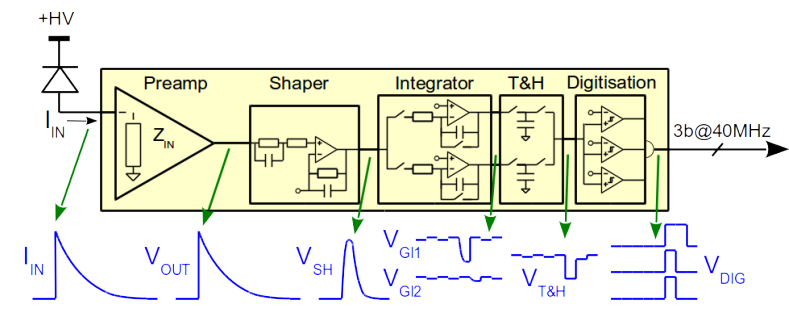




\section{PACIFIC: Preamplifier}

PACIFIC: SiPM Readout

Introduction

\section{PACIFIC}

Preamplifier

Shape

Integrator

Digitizer

Prototypes

PACIFICrO/1

$\mathrm{PACIFICr} 2 / 3$

Outlook

$3 / 15$
- Double feedback current conveyor:

- Fix input voltage and impedance.

- Selectable gains at output mirror.

- Transimpedance amplifier:

- Current to voltage conversion.

- Control conveyor output voltage.

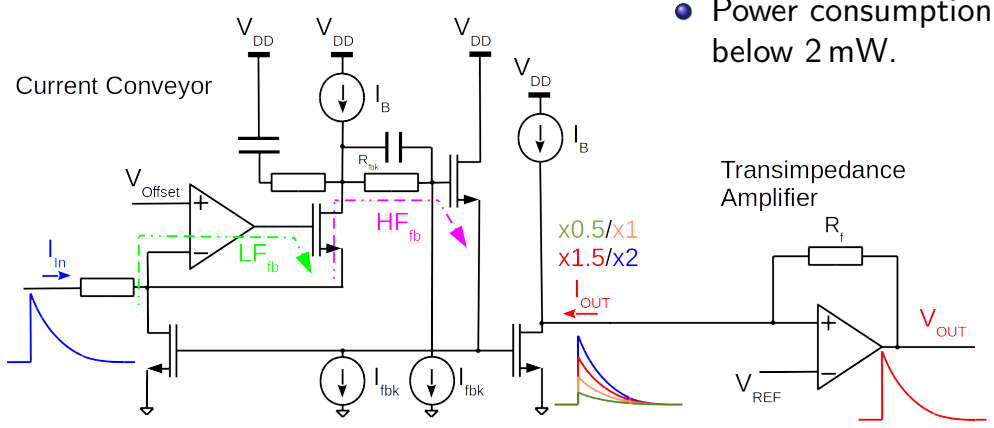

- Bandwidth $250 \mathrm{MHz}$.

- Input impedance $50 \Omega$.

- Input voltage control range $700 \mathrm{mV}$.

- Input dynamic range $4 \mu \mathrm{A}-4 \mathrm{~mA}$.

- Power consumption below $2 \mathrm{~mW}$.

Transimpedance Amplifier 


\section{PACIFIC: Shaper}

PACIFIC

SiPM Readout

Introduction

\section{PACIFIC}

Shaper

Integrator

Digitizer

Prototypes

$\mathrm{PACIFICrO} / 1$

$\mathrm{PACIFICr} 2 / 3$

Outlook
- Double pole-zero cancellation scheme for fast shaping (10 ns).

- Closed-loop OTA circuit with two configurable passive nets:

- First pole-zero net cancels slow component (SiPM capacitance and quenching resistor).

- Second pole-zero net cancels fast component (trace parasitics and input impedance).

- A DC feedback loop controls the quiescent output voltage (critical for the subsequent integration).

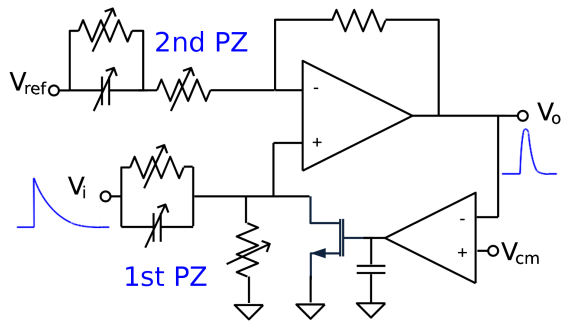

Double PZ Shaper 


\section{PACIFIC: Integrator}

PACIFIC:

SiPM Readout

Introduction

PACIFIC

Preamplifier

Shaper

Integrator

Digitizer

Prototypes

PACIFICrO/1

$\mathrm{PACIFIC} 2 / 3$

Outlook
- Classic closed-loop gated integrator architecture based on a Miller OTA with increased slew rate.

- Dead times avoided by using two interleaved units with independent offset trimming circuits.

- Synchronization with the digitizer is mandatory.

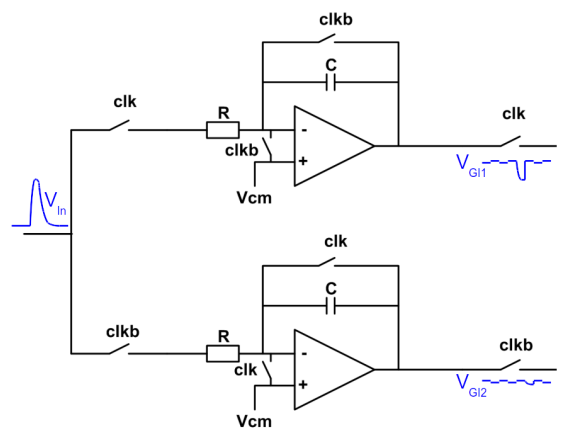

Dual Gated Integrator 


\section{PACIFIC: Digitizer}

PACIFIC

SiPM Readout

Introduction

\section{PACIFIC}

Preamplifier

Shaper

Integrato

Digitizer

Prototypes

$\mathrm{PACIFICrO} /$

$\mathrm{PACIFICr} 2 / 3$

Outlook

- Dual passive track and hold merges the two subchannels.

- Flash ADC using three comparator with:

- Dynamic range: $20-850 \mathrm{mV}$.

- Hysteresis: $10 \mathrm{mV}$.

- Three independent references, configurable per channel, with:

- Dynamic range: $0-750 \mathrm{mV}$.

- Resolution: 8 bits.

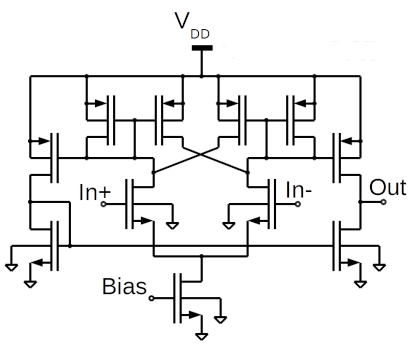

Hysteresis Comparator

- The output of two channels is fed to a serializer that:

- encodes the three comparator outputs into 2 bits.

- streams out both values at $160 \mathrm{MHz}$. 


\section{PACIFICr0 and PACIFICr1}

PACIFIC:

SiPM Readout

Introduction

PACIFIC

Preamplifier

Shaper

Integrator

Digitizer

Prototypes

$\mathrm{PACIFICr0} / 1$

$\mathrm{PACIFICI} 2 / 3$

\section{Outlook}

\section{PACIFICO}

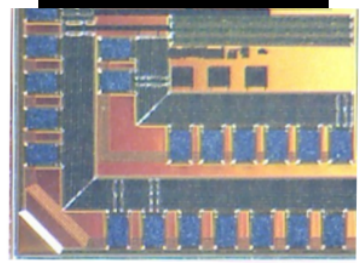

- PACIFICr1:

- November 2013.

- IBM $130 \mathrm{~nm}$.

- One analog FE plus test blocks.

- Analog external bias.

- Independent GI output.
- PACIFICrO:

- May 2013.

- IBM 130 nm.

- Fix gain current conveyor.

- Design migration from AMS 350 nm BiCMOS.

\section{PACIFIC1}

\section{0}

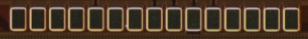




\section{PACIFICr1: Linearity}

PACIFIC: SiPM Readout

Introduction

\section{PACIFIC}

Preamplifier

\section{Shaper}

Integrator

Digitizer

Prototypes $\mathrm{PACIFICr0/1}$ $\mathrm{PACIFICr} 2 / 3$

\section{Outlook}

- Good Preamplifier linearity over full designed dynamic range.

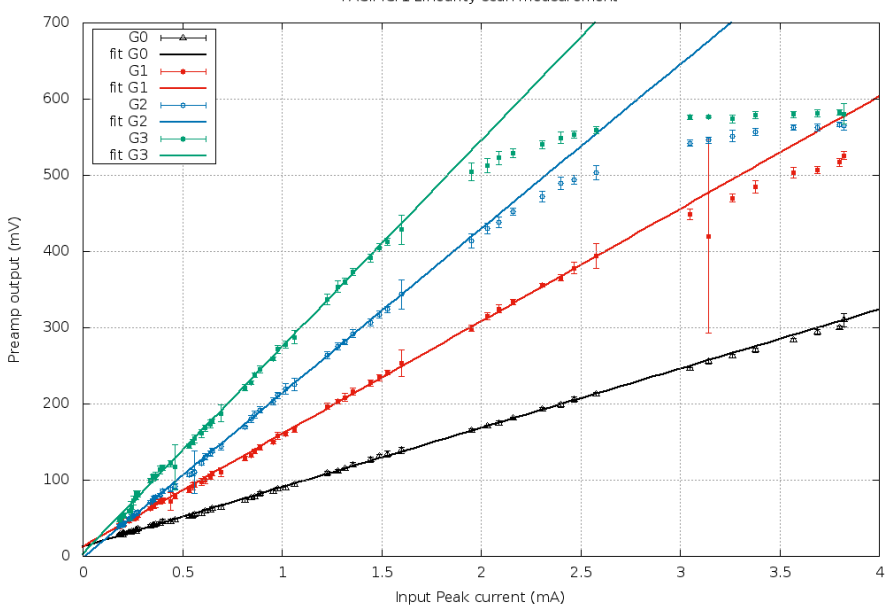




\section{$\mathrm{PACIFICr} 2$ and PACIFICr3}

PACIFIC:

SiPM Readout

Introduction

PACIFIC

Preamplifier

Shaper

Integrator

Digitizer

Prototypes

PACIFICrO/1

$\mathrm{PACIFICr} 2 / 3$

Outlook
- PACIFICr2:

- August 2014.

- IBM $130 \mathrm{~nm}$.

- Eight full FE channels.

- Internal biasing and I2C digital configuration.

\section{PACIFIC2}

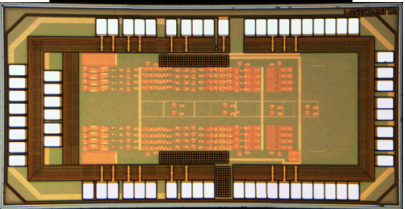

\section{PACIFIC3}

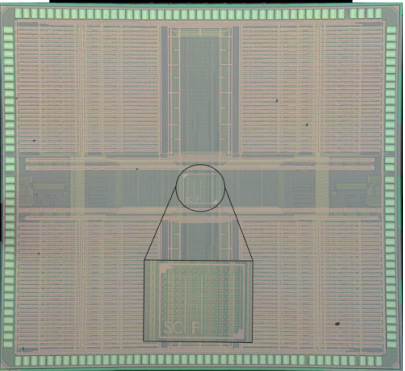

- PACIFICr3:

- July 2015.

- TSMC $130 \mathrm{~nm}$.

- First full size prototype.

- Separate bias left/right. 


\section{PACIFICr3: Power and Bias}

PACIFIC:

SiPM Readout

Introduction

PACIFIC

Preamplifier

Shaper

Integrator

Digitizer

Prototypes

PACIFICrO/1

$\mathrm{PACIFICr} 2 / 3$

\section{Outlook}

$10 / 15$

- Power consumption $\mathbf{1 0 . 4} \mathbf{~ m W} / \mathbf{c h}$, within specifications.

- Bandgap and derived reference voltages slightly low.

- All reference voltage use the same DAC design ( 6 bit) except the threshold of the first comparator (7 bit).

- Reasonably linear response in all measured instances.

PACIFICr3-001-vth1mid

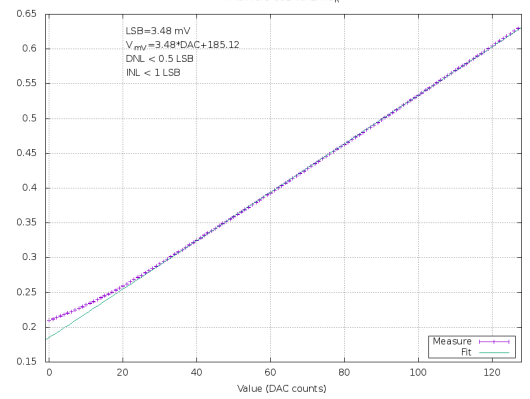

PACIFICr3-OO1-vth2mid

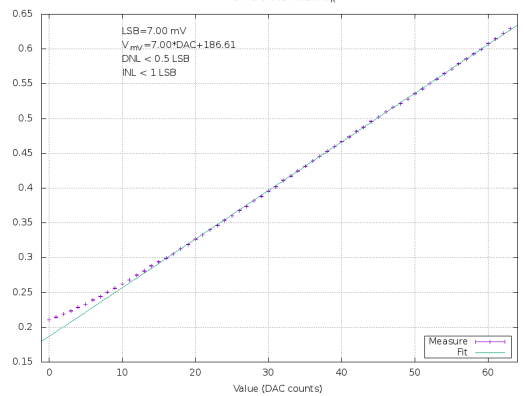




\section{PACIFICr3: Input Voltage}

PACIFIC:

SiPM Readout

Introduction

\section{PACIFIC}

Preamplifier

Shaper

Integrator

Digitizer

Prototypes

PACIFICrO/1

$\mathrm{PACIFICr} 2 / 3$

\section{Outlook}

$11 / 15$
- Derived from a resistor ladder with 16 possible values.

- Range $(700 \mathrm{mV})$ and step $(50 \mathrm{mV})$ as simulated.

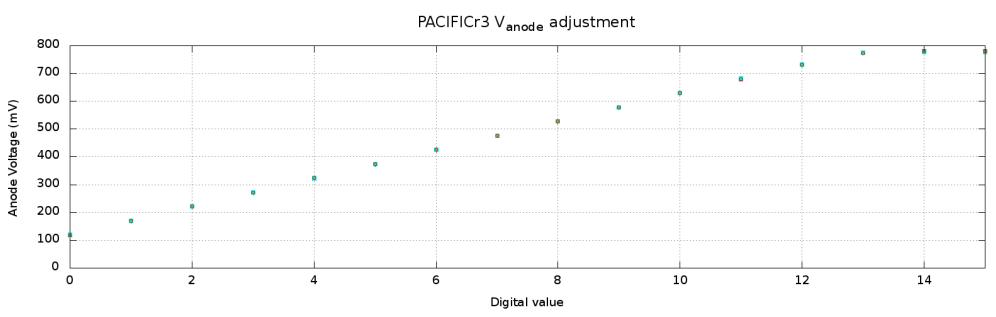

PACIFICr3 $V_{\text {anode }}$ step size

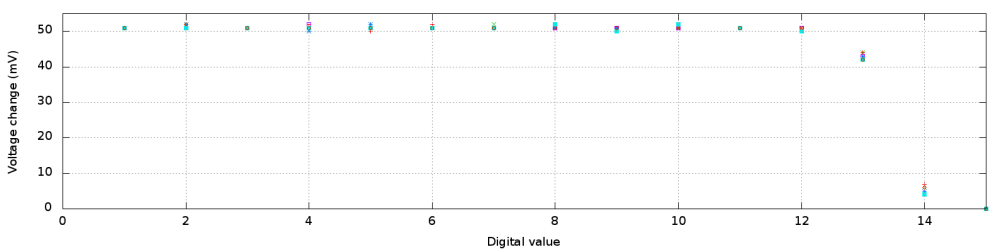




\section{PACIFICr3: Comparator Mismatch}

PACIFIC: SiPM Readout

Introduction

PACIFIC

Preamplifier

Shaper

Integrator

Digitizer

Prototypes

PACIFICrO/1

$\mathrm{PACIFICr} 2 / 3$

Outlook
- Comparator offset voltage obtained using threshold scan.

- Calculated difference to the first comparator offset voltage.

- All channels in two chips evaluated, 256 instances in total.

- Measured $20 \%$ increase w.r.t. Monte Carlo simulations.

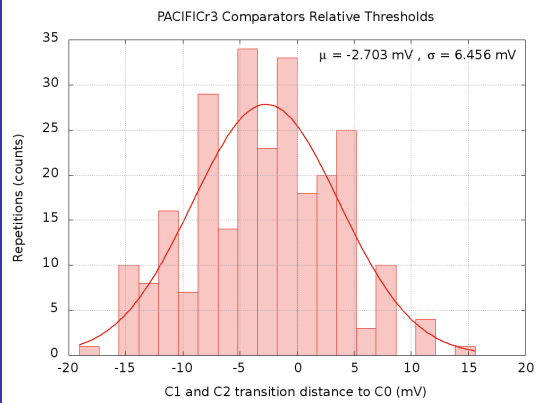




\section{PACIFICr3: Synchronous Light Input}

PACIFIC

SiPM Readout

Introduction

\section{PACIFIC}

Preamplifier

\section{Shaper}

Integrator

Digitizer

Prototypes

PACIFICrO/1

$\mathrm{PACIFICr} 2 / 3$

\section{Outlook}

- Picosecond diode laser synchronously pulsed on SiPM.

- Distinct photoelectron structures are noticeable.

PACIFICr3 Threshold Scan

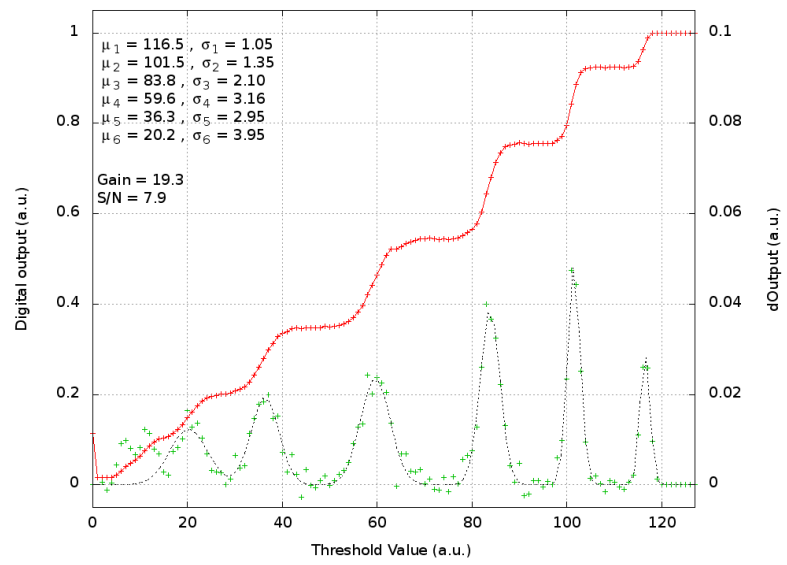




\section{PACIFICr3: Delayed Charge Input}

PACIFIC:

SiPM Readout

Introduction

PACIFIC

Preamplifier

\section{Shaper}

Integrator

Digitizer

Prototypes

PACIFICrO $/ 1$

$\mathrm{PACIFICr} 2 / 3$

\section{Outlook}

- Internal charge injection triggered externally.

- Output sorted by trigger to clock phase difference.

- Response limited by integrator slew rate.

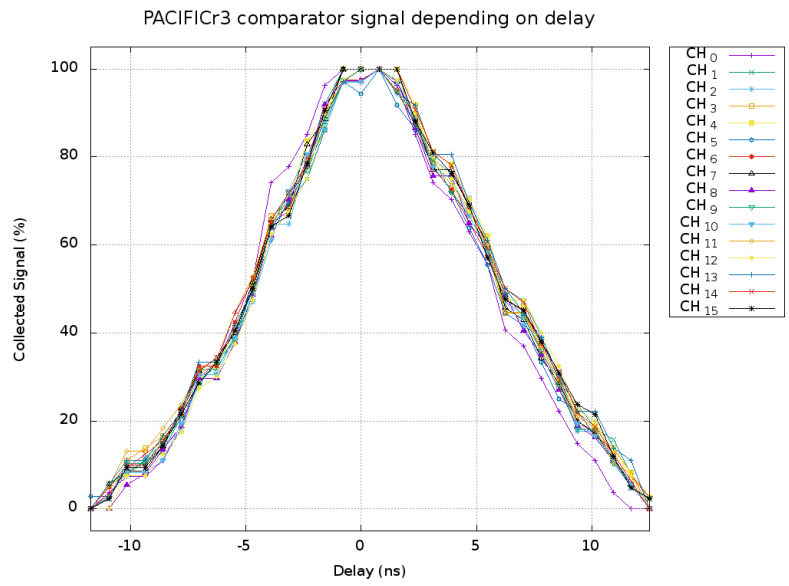




\section{Outlook}

PACIFIC
- PACIFIC is a SiPM readout ASIC with:

- Current input for direct anode connection.

- Fast PZ cancellation shaping for tail suppression.

- Gated integrator damps statistical fluctuations.

- Non-linear configurable 2 bit digital output.

- Channel architecture has been fully validated.

- New prototype PACIFICr4 is in production with:

- Increased slew rate in integrator amplifier.

- Expanded comparator transistor size a factor three, in addition threshold DACs per channel included.

- Custom digital output pads with higher driving power.

- Production readiness review planned for Q2 2017. 
Thanks a lot for your attention! 